\title{
Severely Elevated Fecal Calprotectin in A Pediatric Patient with Persistent Giardiasis
}

\author{
Melissa Shapiro $^{1}$, Neha Shah ${ }^{2}$ and Sona Sehgal ${ }^{1 *}$ \\ ${ }^{1}$ Division of Gastroenterology and Hepatology, Nutrition Children's National Hospital, USA \\ ${ }^{2} J o h n s$ Hopkins Bloomberg School of Public Health, USA
}

*Corresponding author: Sona Sehgal, Division of Gastroenterology and Hepatology, Nutrition Children's National Hospital, Washington, USA.

Received Date: December 15, 2020

Published Date: January 28, 2021

\begin{abstract}
Introduction
Giardia, a protozoan pathogen, is a leading cause of acute gastroenteritis worldwide with higher prevalence in developing countries. It is a treatable diagnosis that should be considered in any patient presenting with clinical symptoms of abdominal pain, bloating, and diarrhea. It is diagnosed with stool microcopy, antigen testing, or immunologic assay. There are few studies on whether Giardia should be considered in the setting of elevated fecal calprotectin (FC), a stool biomarker of intestinal inflammation. We present the case of a patient with markedly elevated FC in the setting of persistent, severe giardiasis.
\end{abstract}

Keywords: Giardia lamblia; Giardiasis; Fecal calprotectin

Abbreviations: FC: Fecal Calprotectin

\section{Introduction}

Giardiasis is a common treatable cause of acute gastroenteritis worldwide, with a prevalence of $2-7 \%$ in high income countries and a higher prevalence in developing countries [1]. It is caused by Giardia lamblia, a flagellated protozoan pathogen that is usually transmitted by fecal-oral route. The life cycle of Giardia includes a cyst form and a trophozoite form. The cyst form is shed into the feces of an infected host and contaminates the environment and fomites. Once ingested, the cysts hatch into trophozoites which actively multiply and attach to the small intestinal wall, causing epithelial inflammation, villous flattening and inhibition of disaccharidases [1]. This results in symptoms of chronic diarrhea, abdominal pain, and weight loss, although some carriers may also be asymptomatic. The gold standard for diagnosing Giardia infection is microscopy, however recent studies suggest molecular detection or immunologic-based assays help increase sensitivity [1]. Other

biomarkers have also been studied in relation to giardiasis. One of these markers is $\mathrm{FC}$, a calcium and zinc binding protein derived primarily from neutrophils [2]. Elevated FC is used as an indicator of intestinal inflammation as it is released into the intestinal lumen during times of leukocyte shedding, cell disturbance, and cell death [2]. It is commonly used in the diagnosis and monitoring of inflammatory bowel disease, but can also be elevated in the setting of polyps and bacterial and viral enteritis $[3,4]$. It is still unclear whether FC elevation can be associated with parasitic infections such as giardiasis. We present a case of markedly elevated FC in a pediatric patient with confirmed giardiasis. Informed patient consent was obtained for publication of the case details.

\section{Case Presentation}

A 6-year-old South Asian female presented to our Gastroenterology clinic with nineteen days of non-bloody, frequent 
diarrhea, nocturnal stooling, daily lower abdominal pain, and a two-pound weight loss. The patient's family also reported two to three days of fever and non-bloody, non-bilious emesis at time of symptom onset. She developed the symptoms while abroad in India and had close contacts with the same symptoms that had selfresolved.

On physical exam, the patient was well-nourished and well hydrated with unremarkable abdominal and perianal exams. Initial laboratory work-up was notable for leukocytosis with WBC $12.46 \mathrm{~K} / \mathrm{mall}$ (lab ref 4.27-11.4 K/mcL), mild thrombocytosis with platelets of $485 \mathrm{~K} / \mathrm{mcL}$ (lab ref 199-367 K/mcL), hypokalemia with potassium of $2.9 \mathrm{mmol} / \mathrm{L}$ (lab ref 3.3-4.7 mmol/L), and mild hypoalbuminemia with albumin $3.5 \mathrm{gm} / \mathrm{dL}$ (lab ref 3.6-5.3 gm/dL). Hemoglobin, hematocrit, erythrocyte sedimentation rate (ESR), C-reactive protein (CRP), IgA level, and tissue transglutaminase IgA (TTG IgA) levels were all normal. Stool studies were positive for giardia and cryptosporidium and FC was elevated at $>2,000 \mathrm{mcg} / \mathrm{g}$ (lab ref for abnormal $>120 \mathrm{mcg} / \mathrm{g}$ ). Giardia cysts were identified on microscopic evaluation of the feces. Stool hemoccult, culture, norovirus, and fecal elastase were normal.

The patient was prescribed metronidazole for treatment of giardia and cryptosporidium, but this was discontinued due to poor patient tolerance. She completed a 3-day course of nitazoxanide with minimal improvement in her symptoms. At this time, her stool was negative for cryptosporidium, but remained positive for giardia antigen with continued elevation of her FC $>2,000 \mathrm{mcg} / \mathrm{g}$. She was then started on a longer, five-day course of nitazoxanide, and subsequently experienced improvement in symptoms. Laboratory follow-up was completed 3 weeks after treatment completion with elevated ESR of $24 \mathrm{~mm} / \mathrm{hr}$ (lab ref $0-20 \mathrm{~mm} / \mathrm{hr}$ ), continued hypoalbuminemia (albumin $3.3 \mathrm{gm} / \mathrm{dL}$ ). Repeat stool studies were obtained over a month after treatment completion and were negative for giardia antigen with improved FC of $338.9 \mathrm{mcg} / \mathrm{g}$. At her most recent visit, the patient had gained two $\mathrm{kg}$ in a month but continued to complain of infrequent mild lower abdominal pain.

\section{Discussion}

FC is an important biomarker for diagnosis and monitoring of inflammatory bowel disease and polyposis, but other diagnoses must also be considered in light of its elevation. Previous research has demonstrated elevated FC levels in bacterial and viral enteritis [4], but there is currently conflicting evidence on its utility in parasitic infections such as giardia. A search for giardia and calprotectin on PubMed yielded nine studies, seven of which were relevant. Four pediatric studies suggested no association between giardia and FC elevation [3,5, 6]. FC has also been studied in puppies with no significant elevation with parasitic infections, including giardia [7]

Conversely, there were also two studies and one case report documenting increased FC levels with persistent or severe giardia infection [8-10]. Unfortunately, these studies did not include the reference range for $\mathrm{FC}$ and did not document the FC levels obtained specifically in Giardiasis patients. Thus, current research suggests that FC may not be significantly elevated in cases of acute, mild giardiasis, but may be elevated in persistent or severe giardiasis. There is lack of evidence on the degree of elevation of FC, even in cases of persistent or severe giardiasis. Interestingly, in our patient's case, her FC was markedly elevated on presentation and improved after adequate treatment of persistent giardiasis, which could suggest that the elevation of her calprotectin was related to the persistent infection. Nonetheless, prolonged patient follow-up and more studies are necessary to elaborate this association. In the meantime, testing for giardia can be considered in the setting of elevated FC in the appropriate clinical context.

\section{Acknowledgement}

None.

\section{Conflict of Interest}

No conflict of interest.

\section{References}

1. Minetti, C, Chalmers RM, Beeching NJ, Probert C, Lamden K (2016) Giardiasis. Bmj 355: i5369.

2. Mumolo MG, Bertani L, Ceccarelli L, Laino G, Di Fluri G, et al. (2018) From bench to bedside: Fecal calprotectin in inflammatory bowel diseases clinical setting. World J Gastroenterol s24(33): 3681-3694.

3. Carroccio A, Iacono G, Cottone M, Di Prima L, Cartabellotta F, et al. (2003) Diagnostic accuracy of fecal calprotectin assay in distinguishing organic causes of chronic diarrhea from irritable bowel syndrome: a prospective study in adults and children. Clin chem 49(6): 861-867.

4. Chen CC, Huang JL, Chang CJ, Kong MS (2012) Fecal calprotectin as a correlative marker in clinical severity of infectious diarrhea and usefulness in evaluating bacterial or viral pathogens in children. J Pediatr Gastroenterol Nutr 55(5): 541-547.

5. Soto-Méndez MJ, Romero-Abal ME, Schümann K, Gil Á, Solomons NW (2017) Normative fecal calprotectin concentrations in Guatemalan preschoolers are high relative to children reported elsewhere. J Pediatr Gastroenterol Nutr 64(2): 238-244.

6. Hestvik E, Tumwine JK, Tylleskar T, Grahnquist L, Ndeezi G, et al. (2011) Faecal calprotectin concentrations in apparently healthy children aged 0-12 years in urban Kampala, Uganda: a community-based survey. BMC pediatr 11(1): 1-7.

7. Grellet A, Heilmann RM, Polack B, Feugier A, Boucraut-Baralon, et al. (2016) Influence of breed size, age, fecal quality, and enteropathogen shedding on fecal calprotectin and immunoglobulin A concentrations in puppies during the weaning period. J Vet Intern Med 30(4): 1056-1064.

8. GOL SMA, Mirjalali H, AGHDAEI HA, Zali MR (2018) Can Giardia Infection Impair the Diagnostic Level of Fecal Calprotectin in Patients with Inflammatory Bowel Disease? A Case Report. Iran J Parasitol 13(3): 505509.

9. Hanevik K, Hausken T, Morken MH, Strand EA, Mørch K, et al. (2007) Persisting symptoms and duodenal inflammation related to Giardia duodenalis infection. J Infect 55(6): 524-530.

10. Versloot CJ, Attia S, Bourdon C, Richardson SE, Potani I, et al. (2018) Intestinal pathogen clearance in children with severe acute malnutrition is unrelated to inpatient morbidity. Clin Nutr ESPEN 24: 109-113. 